\title{
ON THE TOPOLOGICAL CLASSIFICATION OF THE FLOORS OF CERTAIN HILBERT FUNDAMENTAL DOMAINS ${ }^{1}$
}

\author{
MICHAEL H. HALL
}

\begin{abstract}
Associated to the field $Q\left(k^{1 / 2}\right)$ ( $k$ a positive square free integer greater than one), there is a group of transformations of the product of two upper half planes which is analogous to the Hilbert modular group. This group has been shown to have a fundamental domain bounded by a finite number of hypersurfaces. Of particular interest is a subspace of the domain known as the "floor." This floor is a quotient space of a fiber bundle over the circle which is determined by the field $Q\left(k^{1 / 2}\right)$. The principal result of this paper is that, conversely, the topological type (indeed the homotopy type) of this fiber bundle determines the field $Q\left(k^{1 / 2}\right)$ which gives rise to it. This is accomplished by computing the homology groups of the fiber space and showing that the integer $k$ can be determined from these groups.
\end{abstract}

1. Introduction. We consider a generalization of the classical Hilbert modular group which is of interest in certain number theoretic investigations [2], [3], [7]. Let $K=Q\left(k^{1 / 2}\right)(k>1$ and square free) and let $O_{K}$ be the ring of integers in $K$. The group we are interested in is the set of transformations $S$ of the product of two complex upper half planes to itself given by

$$
S\left(z_{1}, z_{2}\right)=\left(\frac{\alpha z_{1}+\beta}{\gamma z_{1}+\delta}, \frac{\alpha^{\prime} z_{2}+\beta^{\prime}}{\gamma^{\prime} z_{2}+\delta^{\prime}}\right)
$$

where $\alpha, \beta, \gamma, \delta$ are in $O_{K}$, the primes denote conjugation, and $\alpha \delta-\gamma \beta=\epsilon$ with $\epsilon$ a totally positive unit in $O_{K}$ (i.e., $\epsilon$ and $\epsilon^{\prime}$ are both positive). It has been shown [1], [7] that this group has a fundamental domain bounded by a finite set of hypersurfaces. In [4] the "floor" of this fundamental domain is defined, and this floor is shown in [6] to be a quotient (under identifications of the group) of a space which is a fiber bundle over the circle $S^{1}$, defined in terms of the field $K$. We shall show that two such spaces are homeomorphic if and only if they arise from the same field.

Received by the editors May 15, 1970.

AMS 1969 subject classifications. Primary 1021, 3265; Secondary 1066.

Key words and phrases. Hilbert modular group, fundamental domain, homology groups, quadratic fields.

1 Some of the results of this paper are contained in the author's dissertation, submitted to the University of Arizona in partial fulfillment of the requirements of the degree of Doctor of Philosophy. 
2. Description of the fiber bundle. Throughout this discussion, $K=Q\left(k^{1 / 2}\right)$ is a quadratic extension of the rationals with $k>1$, square free and such that $K$ has class number 1 . Let $\omega=\left[(c-1)+k^{1 / 2}\right] / c$ with $c=2$ if $k \equiv 1(\bmod 4)$ and $c=1$ otherwise. Then $\{1, \omega\}$ is an integral basis for the ring of integers in $K$ and so, if $\epsilon_{+}$is the fundamental totally positive unit, then $\epsilon_{+}=a+b \omega$ for some rational integers $a$ and $b$. We represent the torus, $T$, as the quotient of the plane by the equivalence relation $E$ under which two points $(x, y)$ and $\left(x^{\prime}, y^{\prime}\right)$ are equivalent if and only if $x-x^{\prime}$ and $y-y^{\prime}$ are both integers. Then any linear transformation of the plane which takes points with integer coordinates to points with integer coordinates induces a map $T \rightarrow T$. With respect to the usual basis in the plane, such a transformation has a matrix whose entries are rational integers. If $\epsilon_{+}=a+b \omega$, the matrix $M_{k}$, given by

$$
\begin{array}{rlr}
M_{k}=\left|\begin{array}{cc}
a & b k \\
b & a
\end{array}\right| & \text { if } k \neq 1(\bmod 4), \\
M_{k}=\left|\begin{array}{cc}
a & b(k-1) / 4 \\
b & a+b
\end{array}\right| & \text { if } k \equiv 1(\bmod 4)
\end{array}
$$

determines a homeomorphism $f_{k}: T \rightarrow T$, since the determinant of $M_{k}$ is 1 (det $M_{k}=$ norm $\epsilon_{+}=1$ ) and so $M_{k}^{-1}$ also has integer entries. The fiber bundle we are interested in is obtained from $[0,1] \times T$ by identifying $(1, x)$ with $\left(0, f_{k}(x)\right)$. It is clear that this gives us the total space of a fiber bundle with base $S^{1}$ and fiber the torus.

3. Homology of bundles over $S^{1}$. Any fiber bundle over $S^{1}$ can be constructed by a generalization of the construction of the preceding section, as follows. We denote the unit interval $[0,1]$ by $I$. Let $X$ be a topological space, $f: X \rightarrow X$ a homeomorphism. In $I \times X$, let $\sim$ be the smallest equivalence relation such that $(1, x) \sim(0, f(x))$. We shall denote by $(X, f)$ the quotient space $(I \times X) / \sim$. In this notation, the space constructed in the preceding section is $\left(T, f_{k}\right)$.

The homology of the spaces we are considering can be computed by applying the next lemma which is a special case of the Wang homology sequence of a fibration over a sphere $[11$, p. 456 , Corollary 6].

Lemma 3.1. Let $Y=(X, f)$, with $X$ compact and Hausdorff. There is a long exact sequence

$$
\cdots \rightarrow H_{i}(X) \rightarrow H_{i}(X) \rightarrow H_{i}(Y) \rightarrow H_{i-1}(X) \rightarrow H_{i-1}(X) \rightarrow \cdots
$$

in which the map $H_{i}(X) \rightarrow H_{i}(X)$ is $f_{*}-1$, the homology homomorphism in dimension $i$ induced by $f$ minus the identity $\operatorname{map} H_{i}(X) \rightarrow H_{i}(X)$. 
For our application, it will be more convenient to use short exact sequences extracted from this long exact sequence.

COROLLARY 3.2. In each dimension, there is a short exact sequence

$$
0 \rightarrow \operatorname{Coker}\left(f_{* i}-1\right) \rightarrow H_{i}(Y) \rightarrow \operatorname{Ker}\left(f_{*(i-1)}-1\right) \rightarrow 0 .
$$

4. The homology of the space $\left(T, f_{k}\right)$ and consequences. In the case we are interested in, the computations are facilitated by the fact that the torus can be given the structure of a finite $\mathrm{C}-\mathrm{W}$ complex and the standard technique of computing the homology of C-W complexes and the homomorphisms induced by maps may be applied (see [8, Chapter V, pp. 173-198] for details). From these considerations, we obtain the homology homomorphisms induced by $f_{k}$. (All homology groups are taken to have integer coefficients.)

LEMMA 4.1. If $M=\left|\begin{array}{ll}a & b \\ c & d\end{array}\right|$ has integer entries and if $f_{M}: T \rightarrow T$ is the map of the torus induced by $M$, then the homology homomorphisms $f_{M *}$ induced by $f_{M}$ are as follows:

(a) In dimension zero, $f_{M *}$ is the identity.

(b) In dimension one, there is a basis for $H_{1}(T)$ such that $f_{M *}$ has matrix $M$ with respect to this basis.

(c) In dimension two, $f_{M *}$ is multiplication by $\operatorname{det} M$.

Specializing to the case of a matrix $M_{k}$, we observe from the definition of the matrix $M_{k}$, that its trace, $\operatorname{tr} M_{k}$, is equal to the trace of $\epsilon_{+}$, and its determinant, $\operatorname{det} M_{k}$, is equal to the norm of $\epsilon_{+}$. Hence $\operatorname{tr} M_{k}=\operatorname{tr} \epsilon_{+}>0$ and $\operatorname{det} M_{k}=1$. If $\epsilon_{+}=a+b \omega$, then since norm $\epsilon_{+}=1$, we have

$$
\begin{aligned}
b^{2} k / 4 & =\left(\left(\operatorname{tr} M_{k}\right) / 2\right)^{2}-1, & & k \equiv 1(\bmod 4) . \\
b^{2} k & =\left(\left(\operatorname{tr} M_{k}\right) / 2\right)^{2}-1, & & k \neq 1(\bmod 4) .
\end{aligned}
$$

THEOREM 4.4. The spaces $\left(T, f_{k}\right),\left(T, f_{k^{\prime}}\right)$ are homeomorphic (in fact, of the same homotopy type) if and only if $k=k^{\prime}$.

Proof. By Corollary 3.2, $H_{1}\left(T, f_{k}\right)=Z \oplus \operatorname{Coker}\left(f_{k * 1}-1\right)$; and, by Lemma $4.1, f_{k * 1}-1$ has matrix $M_{k}-I$. (Here $I$ denotes the identity matrix, and $Z$ the rational integers.) By direct computation, $\operatorname{det}\left(M_{k}-I\right)=2-\operatorname{tr} M_{k}$. Therefore, $\operatorname{det}\left(M_{k}-I\right)=0$ if and only if $\operatorname{tr} M_{k}=2$ but from (4.2) and (4.3) it follows that $\operatorname{tr} M_{k} \neq 2$. Thus $H_{1}\left(\left(T, f_{k}\right)\right)$ is the direct sum of an infinite cyclic group and a torsion group of order $\left|\operatorname{det}\left(M_{k}-I\right)\right|$. Let the order of this torsion group be $n$, so $n=\left|2-\operatorname{tr} M_{k}\right|$, or $\operatorname{tr} M_{k}=2 \pm n$. We know that $\operatorname{tr} M_{k}>0$ and $\operatorname{tr} M_{k} \neq 2$. If $n=1$, i.e., the torsion group is trivial, we have either $\operatorname{tr} M_{k}=1$ or $\operatorname{tr} M_{k}=3$. Examination of 4.2 and 4.3 shows that $\operatorname{tr} M_{k}$ 
$=1$ is impossible, since we must have $b^{2} k>0$ regardless of the choice of $k$, so in this case $\operatorname{tr} M_{k}=3$. Thus we have always $\operatorname{tr} M_{k}=2+n$. Using (4.2) and (4.3) again, we see that if $n$ is odd, then $k$ is the square free part of $4\left(\left(\left(\operatorname{tr} M_{k}\right) / 2\right)^{2}-1\right)$, (and $\left.k \equiv 1(\bmod 4)\right)$. Similarly, if $n$ is even, then $k$ is the square free part of $\left(\left(\operatorname{tr} M_{k}\right) / 2\right)^{2}-1$. Thus $\left(T, f_{k}\right)$, $\left(T, f_{k^{\prime}}\right)$ have the same one dimensional homology if and only if $k=k^{\prime}$, in which case $\left(T, f_{k}\right),\left(T, f_{k^{\prime}}\right)$ are homeomorphic and homotopically equivalent.

REMARK 4.5. In the case $k=5$, the order of the torsion group is 1 , so the homology of the space $\left(T, f_{5}\right)$ is especially uncomplicated. In [5], it is shown that $k=5$ is the only case in which the floor of the fundamental domain is simple, or lies on a single hypersurface.

REMARK 4.6. It is not too surprising that the one dimensional homology group provides enough information to completely determine the spaces we are interested in since, as shown in [10], [12], a class of 3-manifolds, including ours (except perhaps for $k=3$ and 5) is completely classified by the fundamental group.

\section{BIBLIOGRAPHY}

1. O. Blumenthal, Über Modulfunktionen von mehreren Veränderlichen, Math. Ann. 56 (1903), 509-548.

2. H. Cohn, Cusp forms arising from Hilbert's modular functions for the field of $3^{1 / 2}$, Amer. J. Math. 84 (1962), 283-305. MR 26 \#2425.

3. - Some elementary aspects of modular functions in several variables, Bull. Amer. Math. Soc. 71(1965), 681-704. MR 31 \#4769.

4. - On the shape of the fundamental domain of the Hilbert modular group, Proc. Sympos. Pure Math., vol. 8, Amer. Math. Soc., Providence, R.I., 1965, pp. 190202. MR $30 \# 4729$.

5. —, A numerical survey of the floors of various Hilbert fundamental domains, Math. Comp. 19(1965), 594-605. MR 33 \#4016.

6. - A numerical study of topological features of certain Hilbert fundamental domains, Math. Comp. 21(1967), 76-86. MR 36 \#5081.

7. K.-B. Gundlach, Die Bestimmung der Funktionen zur Hilbertschen Modulgruppe des Zahlkörpers $Q(\sqrt{5})$, Math. Ann. 152 (1963), 226-256. MR 29 \#1186.

8. S.-T. Hu, Homology theory: A first course in algebraic topology, Holden-Day, San Francisco, Calif., 1966. MR $36 \# 875$.

9. H. Maass, Über Gruppen von hyperabelschen Transformationen, S.-B. Heidelberger Akad. Wiss. 1940, no. 2. MR 2, 213.

10. L. Neuwirth, $A$ topological classification of certain 3-manifolds, Bull. Amer. Math. Soc. 69(1963), 372-375. MR 26 \#329. 1007.

11. E. H. Spanier, Algebraic topology, McGraw-Hill, New York, 1966. MR 35 \#

12. J. R. Stallings, On fibering certain 3-manifolds, Topology of 3-Manifolds and Related Topics (Proc. Univ. of Georgia Inst., 1961), Prentice-Hall, Englewood Cliffs, N.J., 1962, pp. 95-100. MR $28 \# 1600$.

Texas Tech University, Lubbock, Texas 79409 